\title{
Lieb Mode in a Quasi One-Dimensional Bose-Einstein Condensate of Atoms
}

\author{
A. D. Jackson ${ }^{1}$ and G. M. Kavoulakis ${ }^{2}$ \\ ${ }^{1}$ Niels Bohr Institute, Blegdamsvej 17, DK-2100 Copenhagen Ø, Denmark, \\ ${ }^{2}$ Mathematical Physics, Lund Institute of Technology, P. O. Box 118, S-22100 Lund, Sweden
}

(March 14, 2021)

\begin{abstract}
We calculate the dispersion relation associated with a solitary wave in a quasi-one-dimensional Bose-Einstein condensate of atoms confined in a harmonic, cylindrical trap in the limit of weak and strong interactions. In both cases, the dispersion relation is linear for long wavelength excitations and terminates at the point where the group velocity vanishes. We also calculate the dispersion relation of sound waves in both limits of weak and strong coupling.
\end{abstract}

PACS numbers: 05.45.Y, 03.75.Fi, 05.30.Jp, 67.40.Db

Bose-Einstein condensates of trapped alkali-metal atoms [1] offer a rich source of interesting non-linear phenomena. At mean-field level, the effects of atom-atom interactions can be described as a one-body potential proportional to the local density of atoms. The order parameter, i.e., the condensate wavefunction, then satisfies a non-linear Schrödinger equation which also includes the effect of the confining (harmonic) potential.

Many authors have discussed the properties of solitary waves in a Bose-Einstein condensate of trapped alkalimetal atoms [2 9]. Solitary waves were created and studied experimentally by Burger et al. 10] and by Denschlag et al. [11. Solitary waves result as a balance between the energy cost associated with the Heisenberg principle, $\hbar^{2} / 2 m \xi^{2}$, where $m$ is the atom mass and $\xi$ is the characteristic length of the solitary wave, and the energy gain due to the local density variation of the system, which is of order $n U_{0}$. Here $n$ is the atomic density, and $U_{0}=4 \pi \hbar^{2} a / m$ is the effective two-body interaction matrix element, with $a$ being the scattering length for atomatom collisions. In the present study, we consider repulsive interactions with $a>0$. Equating these two terms, one sees that the characteristic size of a solitary wave is set by the coherence length $\xi$ which satisfies the equation $\hbar^{2} / 2 m \xi^{2}=n U_{0}$. In the experiments of Refs. [10, 11], the effective interaction between the atoms was repulsive, and the solitary waves were thus density depressions. For an attractive effective interaction, the solitary waves are expected to be elevations in the density.

Since the atoms are confined, momentum is not a good quantum number. However, it is possible to use cigarshaped traps which are very long along the $z$ axis. These systems are quasi one-dimensional [5, 12], and the momentum along this axis is approximately a good quantum number.

An interesting question arises in this context. Some 40 years ago, Lieb considered a purely one-dimensional Bose gas of atoms interacting via a contact potential and predicted two distinct modes of excitation [13]. One was identified as the usual Bogoliubov mode. The other class of excitations was later shown by Kulish et al. 114 to be associated with solitary waves (see also Ref. 115.) These authors demonstrated that the dispersion relation result- ing from solitary wave excitation is associated to that predicted by Lieb. It is thus reasonable to ask (at least in the case of quasi one-dimensional atomic condensates) whether this "Lieb mode" exists. Actually, in the recent study of Ref. [16] the Lieb mode was examined in one dimension. Although the theoretical prediction for this mode seems firm, it has never been observed experimentally. In this regard, it is interesting that Stamper-Kurn et al. 17] and Ozeri et al. [18] have recently managed to probe the long wavelength phonon spectrum associated with the Bogoliubov mode in a cigar-shaped condensate of atoms using Bragg spectroscopy. As we argue below, the Lieb mode should be present in such a system and may be observable. Since for long-wavelength excitations the Lieb mode coincides with the usual Bogoliubov mode of sound waves, it is crucial that the momentum imparted to the cloud be appropriately large for the two modes to have distinct energies. One, for example, could excite the cloud using the method of phase imprinting, in order to create a solitary wave, and then measure the excitation energy and the corresponding momentum.

In the present study we derive the dispersion relation associated with the Lieb mode. Reference [19] (and, recently, Ref. [20]) has dealt with the same problem for a different range of parameters using a full threedimensional numerical calculation based on the nonlinear Gross-Pitaevskii equation. In this calculation, the solitary wave is found to be a hybrid between a onedimensional soliton and a three-dimensional vortex ring, but the method is applicable in our limit, as well. Since the present results deal with weaker interactions, we have chosen to adopt a description which neglects the contribution of vortex rings. We distinguish between two limits. In the limit of weak interactions, $n_{0} U_{0} \ll \hbar \omega_{\perp}$, where $n_{0}$ is the maximum density of atoms far away from the wave, and $\omega_{\perp}$ is the frequency of the trapping potential transversely to the long axis of the trap, the resulting equation is the ordinary nonlinear Gross-Pitaevskii equation. In the opposite limit of strong interactions $n_{0} U_{0} \gg \hbar \omega_{\perp}$, the resulting equation is a modified GrossPitaevskii equation, in which the nonlinear term is proportional to the magnitude of the order parameter. Finally, we calculate the usual Bogoliubov mode in both 
regimes of weak and strong interactions and comment on the limits of validity of our study.

Model. We start with a $T=0$ Bose-Einstein condensate of atoms confined in a cylindrical harmonic potential, $V=m \omega_{\perp}^{2}\left(x^{2}+y^{2}\right) / 2$ and assume wave motion along the $z$ axis. There is no confinement along the $z$ axis, and away from the wave, there is a uniform density of atoms, $n(x, y)$, which is independent of $z$. For a short-ranged atom-atom interaction, $V_{\text {int }}\left(\mathbf{r}-\mathbf{r}^{\prime}\right)=U_{0} \delta\left(\mathbf{r}-\mathbf{r}^{\prime}\right)$, the Gross-Pitaevskii equation for the order parameter $\Psi$ has the form

$$
i \hbar \partial_{t} \Psi=\left(-\hbar^{2} \nabla^{2} / 2 m+U_{0}|\Psi|^{2}+V\right) \Psi .
$$

Following Ref. [12], we assume that the transverse dimension of the cloud is sufficiently small and the corresponding time scale sufficiently rapid that the transverse profile of the particle density can adjust to the equilibrium form appropriate for the instantaneous number of particles per unit length. The problem becomes onedimensional, and the solitary pulse can be described by a local velocity, $v(z)$, and a local density of particles per unit length, $\sigma(z)\left[12\right.$ ], $\sigma(z)=\int d x d y|\Psi(x, y, z)|^{2}$. With this assumption, the wavefunction may be written in the form $\Psi(\mathbf{r}, t)=f(z, t) g(x, y, \sigma)$ [5], where $g$ is the equilibrium wavefunction for the transverse motion which depends on time implicitly through the time dependence of $\sigma$. We choose $g$ to be normalized so that $\int|g|^{2} d x d y=1$ and thus, from the equations above, $|f|^{2}=\sigma$.

To proceed, we consider two opposite limits, namely the weak-coupling limit and the Thomas-Fermi regime. The transition between the two limits occurs for $\sigma_{0} a \sim$ $1 / 4$ [5], where $\sigma_{0}$ is the value of $\sigma$ far away from the wave.

Weak-coupling limit. We first consider the weak coupling regime. Although this has traditionally been an academic limit, it is now possible to create Bose-Einstein condensates in cigar-shaped traps 21] which realize the weak-interaction limit. In this case $|g|^{2}$ has a Gaussian form, $|g|^{2}=\left(\pi a_{\perp}^{2}\right)^{-1} e^{-\left(\rho / a_{\perp}\right)^{2}}$. As shown in Ref. [5], $f$ satisfies the equation

$$
i \hbar \partial_{t} f=-\left(\hbar^{2} / 2 m\right) \partial_{z}^{2} f+\hbar \omega_{\perp}\left(1+2 a|f|^{2}\right) f .
$$

We see from this equation that $f \propto e^{-i \omega_{\perp}\left(1+2 a \sigma_{0}\right) t}$ as $|z| \rightarrow \infty$. Thus, we rewrite Eq. (2) using the variable $w=f e^{i \omega_{\perp}\left(1+2 a \sigma_{0}\right) t}$ to obtain

$$
i \hbar \partial_{t} w=-\left(\hbar^{2} / 2 m\right) \partial_{z}^{2} w+\hbar \omega_{\perp} 2 a\left(|w|^{2}-\sigma_{0}\right) w .
$$

Equation (3) has the standard (quadratic) nonlinear term and leads to a speed of sound, $c_{w}$, which satisfies the equation $m c_{w}^{2}=2 \hbar \omega_{\perp} \sigma_{0} a$ 胳] [see also Eq. (15).] Since $\sigma_{0}=n_{0} \pi a_{\perp}^{2}$, we see that $m c_{w}^{2}=n_{0} U_{0} / 2$.

Writing $w=\sqrt{\sigma} e^{i \phi}$ and separating the real and imaginary parts of Eq. (3), we obtain the two hydrodynamic equations

$$
\frac{\hbar^{2}}{2 m}\left(\frac{\partial \sqrt{\sigma}}{\partial z}\right)^{2}=\left(2 \hbar \omega_{\perp} \sigma a-m u^{2}\right) \frac{\left(\sigma-\sigma_{0}\right)^{2}}{2 \sigma},
$$

$$
v=u\left(1-\sigma_{0} / \sigma\right)
$$

Here, we have imposed the boundary condition $v \rightarrow 0$ for $\sigma \rightarrow \sigma_{0}$. The solution of Eq. (4) is

$$
\sigma(z) / \sigma_{0}-1=-\frac{\cos ^{2} \theta}{\cosh ^{2}(z \cos \theta / \zeta)},
$$

where $\theta=\arcsin \left(u / c_{w}\right)$ and $\zeta=2 \xi\left(n_{0}\right)$ with $\xi\left(n_{0}\right)$ equal to the coherence length for $n_{0}=\sigma_{0} /\left(\pi a_{\perp}^{2}\right)$ (i.e., $\zeta=$ $\left.a_{\perp} /\left(2 \sigma_{0} a\right)^{1 / 2}\right)$. The wavefunction $w$ can also be written as

$$
w=\sqrt{\sigma_{0}}[i \sin \theta+\cos \theta \tanh (z \cos \theta / \zeta)] .
$$

Energy and momentum of the solitary wave. In the limit of weak interactions, Eq. (3) implies that

$$
\begin{array}{r}
\mathcal{E}=\int\left(\frac{\hbar^{2}}{2 m} \frac{\partial w^{*}}{\partial z} \frac{\partial w}{\partial z}+\hbar \omega_{\perp} a\left(w w^{*}\right)^{2}-2 \hbar \omega_{\perp} a \sigma_{0} w w^{*}\right. \\
\left.+\hbar \omega_{\perp} a \sigma_{0}^{2}\right) d z
\end{array}
$$

where the final term, which represents the energy of the background density of atoms, ensures convergence of the integral. Equation (8) can be written as

$$
\begin{array}{r}
\mathcal{E}=\int\left[\frac{\hbar^{2}}{2 m}\left(\frac{\partial \sqrt{\sigma}}{\partial z}\right)^{2}+\frac{\hbar^{2} \sigma}{2 m}\left(\frac{\partial \phi}{\partial z}\right)^{2}\right. \\
\left.+\hbar \omega_{\perp} a\left(\sigma-\sigma_{0}\right)^{2}\right] d z .
\end{array}
$$

Since $v=\hbar \partial_{z} \phi / m$, Eqs. (4) and (5) allow us to reduce Eq. (9) to the simpler form

$$
\mathcal{E}=2 \hbar \omega_{\perp} a \int\left(\sigma-\sigma_{0}\right)^{2} d z .
$$

Given the solitary wave profile of Eq. (6), Eq. (10) yields

$$
\mathcal{E}=(4 \sqrt{2} / 3) \mathcal{E}_{0} \cos ^{3} \theta,
$$

where $\mathcal{E}_{0}=\hbar \omega_{\perp}\left(\sigma_{0} a\right)^{1 / 2} \sigma_{0} a_{\perp}$.

Calculation of the momentum $\mathcal{P}$,

$$
\mathcal{P}=-i \hbar \int w^{*} \frac{\partial w}{\partial z} d z=m \int \sigma(z) v(z) d z
$$

requires a more careful description of the solitary-wave profile for large $|z|$. This is most easily accomplished by requiring that the solitonic wavefunction is periodic on a large interval $[-L / 2,+L / 2]$. The solution to this problem for all $L$ can be expressed analytically in terms of Jacobi elliptic functions 22]. For our purposes, it is sufficient to note the behaviour of $\sigma$ and $\phi$ in the limit of large $L$. For $z \sim \mathcal{O}\left(L^{0}\right), \sigma$ is still given by Eqs. (6) and (7). For positive $u$, Eq. (5) indicates that $\partial_{z} \phi$ is negative and that a net phase will accumulate as we move from the center of the solitary wave at $z=0$ towards $z=+L / 2$. For $z \sim \mathcal{O}(L)$, however, the periodic $\sigma$ approaches a constant value larger than $\sigma_{0}$ by an amount proportional 
to $1 / L$. In this region, $\partial_{z} \phi$ is positive, the total phase accumulated between $z=0$ and $+L / 2$ is precisely 0 , and the periodic boundary conditions are fulfilled. Evidently, this large- $z$ behaviour makes a finite contribution to the momentum in the $L \rightarrow \infty$ limit which is readily evaluated from Eq. 12). To leading order, the momentum can be evaluated using Eqs. (6) and (7) to obtain

$$
\mathcal{P}=m \int_{-\infty}^{\infty}\left(\sigma-\sigma_{0}\right) v(z) d z=m u \int_{-\infty}^{\infty} \frac{\left(\sigma-\sigma_{0}\right)^{2}}{\sigma} d z
$$

Using the order parameter of Eq. (耳), we find that

$$
\mathcal{P}=\mathcal{P}_{0}(\pi u /|u|-2 \theta-\sin 2 \theta)
$$

where $\mathcal{P}_{0}=\sigma_{0} \hbar$. The momentum $\mathcal{P}$ was also determined in Ref. [15 using the macroscopic relation $\mathcal{P}=\int u^{-1}(\partial \mathcal{E} / \partial u) d u$. This yields a result identical to Eq. (14). Equation (14) implies that a maximum momentum of $\mathcal{P}_{\max }=\pi \sigma_{0} \hbar$ is obtained for $u=0$. Note that, to leading order in $1 / L$, the energy of Eq. (11) is unaltered by the imposition of periodic boundary conditions.

Combining Eqs. (11) and (14), we arrive at the dispersion relation $\mathcal{E}(\mathcal{P})$ for the Lieb mode in the limit of weak interactions. The solid line of Fig. 1(a) shows this result. For $\mathcal{P} \rightarrow 0, \mathcal{E}=c_{w} \mathcal{P}$, in agreement with the usual Bogoliubov dispersion relation discussed below. The Lieb mode terminates at $\mathcal{P}=\mathcal{P}_{\max }$ where it has an energy of $\mathcal{E} / \mathcal{E}_{0}=4 \sqrt{2} / 3$.

Bogoliubov mode in the weak interaction limit. Equation (3) implies that the Bogoliubov mode obeys the dispersion relation

$$
\frac{\mathcal{E}}{\mathcal{E}_{0}}=\sqrt{2} \frac{|\mathcal{P}|}{\mathcal{P}_{0}} \sqrt{1+\left(\frac{\mathcal{P}}{\mathcal{P}_{0}}\right)^{2} \frac{\sigma_{0} a}{8}\left(\frac{a_{\perp}}{a}\right)^{2}}
$$

Choosing $\sigma_{0} a=0.1, a_{\perp}=1 \mu \mathrm{m}$, and $a=100 \AA$, the coefficient inside the square root is 125 . This number is relatively large because the characteristic wavevector corresponding to $\mathcal{P}_{0}$, i.e., $\sigma_{0}$, is much larger than the inverse coherence length, $\xi^{-1}\left(n_{0}\right)$. Specifically, $\xi^{-1}\left(n_{0}\right) \sim$ $\left(8 \sigma_{0} a\right)^{1 / 2} / a_{\perp}$. Thus, $\sigma_{0} \xi\left(n_{0}\right) \sim a_{\perp}\left(\sigma_{0} / a\right)^{1 / 2} \sim 10$. The dotted line in Fig. 1(a) shows the dispersion relation of Eq. (15) for the above numbers.

The strong coupling limit. In the limit of strong interactions, $n_{0} U_{0} \gg \hbar \omega_{\perp}$, we use the Thomas-Fermi approximation for the transverse profile, $|g|^{2}=2\left(\pi R_{\perp}^{2}\right)^{-1}(1-$ $\left.\rho^{2} / R_{\perp}^{2}\right)$, with $R_{\perp} / a_{\perp}=2(\sigma a)^{1 / 4}$ [12]. From Ref. [5] we again see that $w=f e^{-2 i \omega_{\perp}\left(\sigma_{0} a\right) t}$ satisfies the equation

$$
i \hbar \partial_{t} w=-\left(\hbar^{2} / 2 m\right) \partial_{z}^{2} w+2 \hbar \omega_{\perp} a^{1 / 2}\left(|w|-\left|w_{0}\right|\right) w .
$$

The effective equation obeyed by $w$ now involves a modified non-linear term proportional to $|w|$. Equation (16) implies a sound speed of $m c_{s}^{2}=\hbar \omega_{\perp}\left(\sigma_{0} a\right)^{1 / 2}$ (see also Eq. (22).] Since $n_{0} U_{0}=2 \hbar \omega_{\perp}\left(\sigma_{0} a\right)^{1 / 2}$, this becomes $m c_{s}^{2}=n_{0} U_{0} / 2$ [5. 12$]$.
Using Eq. 16), we again write $w=\sqrt{\sigma} e^{i \phi}$ and separate real and imaginary parts. The velocity is still given by Eq. (5). In addition, Eq. (16) implies that

$$
\begin{aligned}
\frac{\hbar^{2}}{2 m}\left(\frac{\partial \sqrt{\sigma}}{\partial z}\right)^{2}=\frac{2}{3} \hbar \omega_{\perp} a^{1 / 2}\left(2 \sigma^{3 / 2}\right. & \left.-3 \sigma_{0}^{1 / 2} \sigma+\sigma_{0}^{3 / 2}\right) \\
& -m u^{2} \frac{\left(\sigma-\sigma_{0}\right)^{2}}{2 \sigma} .
\end{aligned}
$$

This equation provides a relation between $u$ and the minimum value of $\sigma, \sigma_{\min }$. For a given $u, \sigma_{\min }$ is given by the non-trivial root $\left(\sigma \neq \sigma_{0}\right)$ of the right side of Eq. (17).

Energy and momentum in the strong interaction limit. Using Eq. (13) we find that the momentum is given by

$$
\mathcal{P} / \mathcal{P}_{0}=u / c_{s} \int(y-1)^{2} / y d x,
$$

where $x=z\left(\sigma_{0} a\right)^{1 / 4} / a_{\perp}$ and $y=\sigma / \sigma_{0}$. Here, $\sigma$ is the solution of Eq. (17). In addition, Eq. (16) gives an energy

$$
\begin{aligned}
\mathcal{E}=\int & {\left[\frac{\hbar^{2}}{2 m}\left(\frac{\partial \sqrt{\sigma}}{\partial z}\right)^{2}+\frac{\sigma \hbar^{2}}{2 m}\left(\frac{\partial \phi}{\partial z}\right)^{2}+\right.} \\
& \left.+\frac{2}{3} \hbar \omega_{\perp} a^{1 / 2}\left(2 \sigma^{3 / 2}-3 \sigma \sigma_{0}^{1 / 2}+\sigma_{0}^{3 / 2}\right)\right] d z .
\end{aligned}
$$

The final term in the integral again guarantees its convergence and corresponds to the energy of the background density.

Since $v=\hbar \partial_{z} \phi / m$, Eq. (17) and the formula $v=u(1-$ $\left.\sigma_{0} / \sigma\right)$ allow us to write Eq. (19) as

$$
\mathcal{E}=(4 / 3) \hbar \omega_{\perp} a^{1 / 2} \int\left(2 \sigma^{3 / 2}-3 \sigma \sigma_{0}^{1 / 2}+\sigma_{0}^{3 / 2}\right) d z .
$$

Introducing the unit of energy $\mathcal{E}_{0}^{\prime}=\hbar \omega_{\perp}\left(\sigma_{0} a\right)^{1 / 4} \sigma_{0} a_{\perp}$,

$$
\mathcal{E} / \mathcal{E}_{0}^{\prime}=\frac{4}{3} \int\left(2 y^{3 / 2}-3 y+1\right) d x .
$$

We have solved Eq. (17) numerically to obtain $\sigma(z)$ for various values of $u$. This numerical solution was used in Eqs. (18) and (21) to determine $\mathcal{P}(u)$ and $\mathcal{E}(u)$, respectively. The solid line in Fig. 1(b) shows the resulting dispersion relation for the Lieb mode in the limit of strong interactions. The $x$ axis is measured in units of $\mathcal{P}_{0}$, and the $y$ axis is measured in units of $\mathcal{E}_{0}^{\prime}$. As in the case of weak interactions, the slope of the curve for small values of $\mathcal{P}$ is equal to $c_{s}$, and also the curve terminates at $\pi \mathcal{P}_{0}$ with $\mathcal{E} \approx 1.5 \mathcal{E}_{0}^{\prime}$.

Bogoliubov mode in the limit of strong interactions. Equation (16) implies that the dispersion relation associated with the Bogoliubov mode is

$$
\frac{\mathcal{E}}{\mathcal{E}_{0}^{\prime}}=\frac{|\mathcal{P}|}{\mathcal{P}_{0}} \sqrt{1+\left(\frac{\mathcal{P}}{\mathcal{P}_{0}}\right)^{2} \frac{1}{4}\left(\sigma_{0} a\right)^{3 / 2}\left(\frac{a_{\perp}}{a}\right)^{2}} .
$$

For $\sigma_{0} a=1, a_{\perp}=1 \mu m$, and $a=100 \AA$, the coefficient inside the square root is $\approx 2500$, which is $\gg 1$, since the 
characteristic wavevector corresponding to $\mathcal{P}_{0}$, i.e., $\sigma_{0}$, is $\gg$ than $\xi^{-1}\left(n_{0}\right): \sigma_{0} \xi\left(n_{0}\right) \sim \sigma_{0} a_{\perp} /\left(\sigma_{0} a\right)^{1 / 4}$. The dotted line in Fig. 1(b) shows the dispersion relation of Eq. (22) for this choice of parameters. Once again, the Lieb and the Bogoliubov modes have identical dispersion relations in the limit of long wavelength excitations, as expected.

The methods adopted here are expected to be reliable for weak interactions [5] for which the width of the solitary wave is much larger than the transverse width of the cloud. In this regime the transverse degrees of freedom are frozen out, and the behavior of the system is essentially one-dimensional. As the strength of the interaction is increased, it has been demonstrated in Ref. [6] that the dark solitary waves become unstable. (See also Ref. [8].) As shown in this reference, dark solitary waves in a cylindrical trap become unstable for $n_{0} U_{0} / \hbar \omega_{\perp} \geq 2.4$. Assuming that the system is in the Thomas-Fermi regime, $\sigma_{0} /\left(\pi R_{\perp}^{2}\right)=n_{0} / 2$, with $R_{\perp}^{2}=4 a_{\perp}^{2}\left(\sigma_{0} a\right)^{1 / 2}[12]$, and therefore the corresponding critical value of $\sigma_{0} a$ is $\approx 1.2^{2}=1.44$. Thus, our approach should also provide a reasonable variational description of the dispersion relation in the strong interaction regime provided only that dark solitary waves are stable. In addition, we note that the Bogoliubov dispersion relation obtained here is in analytic agreement with numerical solutions 23] in the large and small momentum limits for both weak and strong interactions. Differences of only a few percent for intermediate momenta are found for $\sigma_{0} a$ as large as 1.

In summary, we have calculated the dispersion relation $\mathcal{E}=\mathcal{E}(\mathcal{P})$ of a sound wave and of a solitary wave in a quasi one-dimensional Bose-Einstein condensate of atoms confined in a harmonic, cylindrical trap in the limits of weak and strong interactions. For solitary waves, in both limits the spectrum has the same qualitative behaviour: it is linear for long wavelength excitations and coincides with the Bogoliubov mode. For shorter wavelength excitations, it lies below the Bogoliubov mode, and it terminates at a maximum momentum for which the group velocity vanishes.

We would like to thank N. Papanicolaou for pointing out the existence of the Lieb mode to us and for useful discussions. We also acknowledge profitable discussions with C. J. Pethick. G.M.K. was supported by the Swedish Research Council (VR), and by the Swedish Foundation for Strategic Research (SSF). G.M.K. would like to thank the Physics Dept. of the Univ. of Crete for its hospitality.

[1] C. J. Pethick and H. Smith, Bose Einstein Condensation in Dilute Gases (Cambridge University Press, Cambridge, England, 2001).

[2] S. A. Morgan et al., Phys. Rev. A 55, 4338 (1997).
[3] T. F. Scott et al., J. Phys. B 31, L329 (1998).

[4] R. Dum et al., Phys. Rev. Lett. 80, 2972 (1998).

[5] A. D. Jackson et al., Phys. Rev. A 58, 2417 (1998).

[6] A. E. Muryshev et al., Phys. Rev. A 60, R2265 (1999).

[7] T. Busch and J. R. Anglin, Phys. Rev. Lett. 84, 2298 (2000).

[8] D. L. Feder et al., Phys. Rev. A 62, 053606 (2000).

[9] B. Wu et al., e-print cond-mat/0105377.

[10] S. Burger et al., Phys. Rev. Lett. 83, 5198 (1999).

[11] J. Denschlag et al., Science 287, 97 (2000).

[12] G. M. Kavoulakis and C. J. Pethick, Phys. Rev. A 58, 1563 (1998).

[13] E. Lieb, Phys. Rev. 130, 1616 (1963).

[14] P. P. Kulish et al., Theor. Math. Phys. 28, 615 (1976).

[15] M. Ishikawa and H. Takayama, J. Phys. Soc. Japan 49, 1242 (1980).

[16] E. B. Kolomeisky et al., Phys. Rev. Lett. 85, 1146 (2000).

[17] D. M. Stamper-Kurn et al., Phys. Rev. Lett. 83, 2876 (1999).

[18] R. Ozeri et al., e-print cond-mat/0112496.

[19] S. Komineas and N. Papanicolaou, e-print condmat/0202182.

[20] S. Komineas and N. Papanicolaou, e-print condmat/0204136.

[21] A. Görlitz et al., Phys. Rev. Lett. 87, 130402 (2001).

[22] T. Tsuzuki, J. Low Temp. Phys. 4, 441 (1971).

[23] P. O. Fedichev and G. V. Shlyapnikov, Phys. Rev. A 63, 045601 (2001).
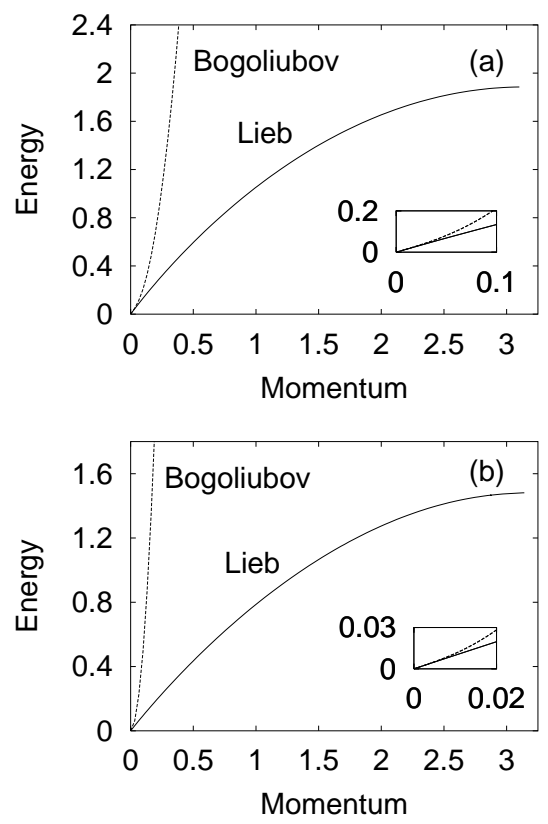

FIG. 1. (a) Dispersion relation $\mathcal{E}=\mathcal{E}(\mathcal{P})$ in the limit of weak interactions for a solitary wave (solid curve) and for the Bogoliubov mode (dotted line.) The energy is measured in units of $\mathcal{E}_{0}$ and the momentum in units of $\mathcal{P}_{0}$. (b) Same as (a) in the limit of strong interactions, with the energy measured in units of $\mathcal{E}_{0}^{\prime}$. The inset shows the same graph on a smaller scale, for long-wavelength excitations. 